\title{
Infection Prophylaxis and Management
}

National Cancer Institute

\section{Source}

National Cancer Institute. Infection Prophylaxis and Management. NCI Thesaurus. Code C15511.

Treat infections with antibacterial, antifung al, antiparasite or antiviral drugs 